\title{
LOWER BOUNDS FOR THE DOMINATION NUMBER
}

\author{
ERMELINDA DELAVIÑA, RYAN PEPPER, AND BILL WALLER \\ UNIVERSITY OF HOUSTON - DOWNTOWN, HOUSTON, TX, 77002
}

\begin{abstract}
In this note, we prove several lower bounds on the domination number of simple connected graphs. Among these are the following: the domination number is at least two-thirds of the radius of the graph, three times the domination number is at least two more than the number of cut-vertices in the graph, and the domination number of a tree is at least as large as the minimum order of a maximal matching.
\end{abstract}

\section{Introduction and Key Definitions}

Let $G=(V, E)$ be a simple connected graph of finite order $n=|V|$. Although we may identify a graph $G$ with its set of vertices, in cases where we need to be explicit we write $V(G)$ to denote the vertex set of $G$. A set $D$ of vertices of a graph $G$ is called a dominating set provided each vertex of $V-D$ is adjacent to a member of $D$. The domination number of $G$, denoted $\gamma=\gamma(G)$, is the cardinality of a smallest dominating set in $G$. The eccentricity of a vertex $v \in G$ is the maximum of distances from $v$ to any of the other vertices of $G$ - where the distance between two vertices means the number of edges in a shortest path connecting them. The minimum eccentricity of the graph is called the radius and denoted $r(G)$. The maximum eccentricity of the graph is called the diameter and denoted $d(G)$. A vertex of minimum eccentricity is called a center vertex of $G$ and the center set $C(G)$ (or center) is the set of all centers of the graph. The eccentricity of the center of $G$, denoted by $\hat{r}(G)$, is the maximum distance from the center set to vertices not in the center set, where the distance from a vertex to a set is the smallest distance from the vertex to any of the vertices in the set. A vertex of maximum eccentricity is called a boundary vertex of $G$ and the boundary set $B(G)$ (or boundary) is the set of all boundary vertices of the graph. Vertices of maximum eccentricity are also called peripheral and what we call the boundary is also called the periphery [2]. The eccentricity of the boundary of $G$, denoted by $\hat{d}(G)$, is the maximum distance from the boundary set to vertices not in the boundary set. Finally, a matching is an independent set 
of edges and a cut-vertex is a vertex whose deletion increases the number of components. For general graph theory terminology and notation that is not presented here, the reader can refer to [1] or [23].

Before moving on, it is helpful to observe the following basic properties of the distance invariants mentioned above.

Proposition 1. For any connected graph $G$,

(i) $r(G) \leq d(G) \leq 2 r(G)$;

(ii) $\hat{r}(G) \leq r(G)$;

(iii) $\hat{d}(G) \leq d(G)-1$

The first of these is easy and well known. The second is fairly obvious. For the third, let $v$ be a vertex not in the boundary that realizes the maximum distance to the boundary. If this distance is at least $d(G)$, then it is exactly $d(G)$ by definition of diameter. Consequently, $v$ is a vertex of maximum eccentricity and is thus in the boundary, which is a contradiction.

The domination number is one of the most studied simple graph invariants. Indeed, there have been at least two books $([18,19])$ written on this invariant alone. It is the aim of this paper to prove several new lower bounds on the domination number of graphs. A few of these theorems were inspired by conjectures of the computer program Graffiti.pc, written by E. DeLaViña. In addition, all of these results have analogs for total domination (see $[3,8]$ ). For example, in $[8]$ it is proven that the total domination number of a graph is at least the radius of the graph.

\section{LEMMAS}

We start by stating a couple of lemmas that will be referred to several times in the main section of the paper.

Given a graph $G$ with dominating set $D$, a vertex $v \notin D$ is over-dominated by $D$ if it has two or more neighbors in $D$. The over-domination number of $v$ with respect to $D$, denoted by $O_{D}(v)$, is one less than the number of neighbors $v$ has in $D$.

Lemma 2. Let $T$ be a tree and let $D$ be a minimum dominating set of $T$ with $k$ components. Denote the number of edges both of whose vertices are in $D$ by $e_{1}$, the number of edges both of whose vertices are in $H=T-D$ by $e_{2}$, and the number of edges with one vertex in $D$ and the other vertex in $H=T-D$ by $e_{3}$. Moreover, let $j$ be the number of 
components of $H=T-D$ with at least two neighbors in $D$ (the non-trivial components of $H$ ) and let $l_{H}$ be the number of components of $H=T-D$ with exactly one neighbor in $D$ (the leaves of $H$ ). Then all of the following are true:
a) $e_{1}=|D|-k$
b) $e_{2}=k-1-\sum_{v \in H} O_{D}(v) \leq k-1$
c) $e_{3}=n-|D|+\sum_{v \in H} O_{D}(v) \geq n-|D|$
d) $2 j+l_{H} \leq e_{3}=k+j+l_{H}-1$
e) $n-l_{H}+2+\sum_{v \in H} O_{D}(v) \leq 2 k+|D|$.

A simple proof of the above lemma was given in [9]. The lemma below appears to be folklore (its proof is very similar to the proof of Lemma 2 in [8]).

Lemma 3. Let $G$ be a connected graph, with $n>1$. Then for any minimum dominating set $D$ of $G$, there is a spanning tree $T$ of $G$ such that $D$ is a minimum dominating set of $T$.

\section{TheOREMS}

The following elementary result is a rediscovery, and can be obtained quite easily, as is shown in [18]. One of our main results, Theorem 5, is an improvement on this result anytime the diameter is strictly less than twice the radius.

Theorem 4. Let $G$ be a connected graph with $n>1$ and diameter d. Then,

$$
\gamma \geq \frac{d+1}{3}
$$

Theorem 5 below is a near generalization of Theorem 4. Indeed, since the diameter can actually equal the radius, it is sometimes twice as good as a lower bound on the domination number (take cycles for instance). Moreover, it is similar to the well known result that the independence number is at least the radius - originally a conjecture of Graffiti [13] and proven independently several times $[11,12,14,15]$. In addition, it is shown in [8] that the total domination number (that is, the cardinality of a set of minimum order having the property that every vertex in the graph is adjacent to a vertex in the set) is at least the radius.

Theorem 5. Let $G$ be a connected graph with $n>1$. Then,

$$
\gamma \geq \frac{2}{3} r .
$$


Moreover, this bound is sharp.

Proof. Let $D$ be a minimum dominating set of $G$. Form a spanning tree $T$ of $G$, as prescribed in Lemma 3, so that $D$ is also a minimum dominating set of $T$. Since $r(G) \leq$ $r(T), 2 r(T)-1 \leq d(T)$ (because $T$ is a tree) and $\gamma(T)=\gamma(G)$ (by Lemma 2), we can apply Theorem 4 to $T$ and obtain the following chain of inequalities:

$$
2 r(G)-1 \leq 2 r(T)-1 \leq d(T) \leq 3 \gamma(T)-1=3 \gamma(G)-1
$$

Equality holds in the bound above for cycles with orders congruent to 0 modulo 6 . On the other hand, the tree obtained by amalgamating a pendant vertex to each vertex of a path has radius about $\frac{n}{3}$ while it has domination number of $\frac{n}{2}$ - thus showing that the difference between these two expressions can be made arbitrarily large.

The following theorem, proven by Lemańska in [20], follows directly from part e) of Lemma 2.

Theorem 6. If $T$ is a tree with $l$ leaves, then

$$
\gamma \geq \frac{n-l+2}{3}
$$

We can use Theorem 6 together with Lemma 3 to deduce the more general result below, which is equivalent to Theorem 6 for trees.

Theorem 7. For any connected graph $G$ with $x$ cut-vertices,

$$
\gamma \geq \frac{x+2}{3}
$$

Moreover, this bound is sharp.

Proof. Let $D$ be a minimum dominating set of $G$. Form a spanning tree $T$ of $G$, as prescribed in Lemma 3, so that $D$ is also a minimum dominating set of $T$. Let $x(T)$ denote the number of cut-vertices of $T$ and note that $x(T) \geq x$, since any cut-vertex of $G$ is also a cut-vertex of $T$. Now, applying Theorem 6 to $T$ we find,

$$
\gamma(G)=\gamma(T) \geq \frac{n-l(T)+2}{3}=\frac{x(T)+2}{3} \geq \frac{x+2}{3} .
$$


Lemanska shows in [20] that equality holds in Theorem 6 if and only if $T$ is a tree such that the distance between any two leaves is congruent to 2 modulo 3. Since for trees, the number of cut-vertices is exactly $n-l$, equality holding in Theorem 6 is a sufficient condition for equality holding in the above theorem. An example of a graph where equality holds in Theorem 7 that is not necessarily a tree is a graph with a cut-vertex of degree $n-1$. On the other hand, since cycles have no cut-vertices, the difference between the expressions in Theorem 7 can be made arbitrarily large.

The next theorem is a very slight improvement on Theorem 5 whenever $r=\hat{r}$. For instance, in graphs with a unique center vertex, such as odd paths, $r=\hat{r}$ and the lower bound below is $\frac{1}{3}$ more than that achieved in Theorem 5 .

Theorem 8. Let $G$ be a connected graph with $n>1$. Then,

$$
\gamma \geq \frac{2}{3} \hat{r}(G)+\frac{1}{3}
$$

Moreover, this bound is sharp.

Proof. Let $D$ be a minimum dominating set of $G$. Form a spanning tree $T$ of $G$, as prescribed in Lemma 3 , so that $D$ is also a minimum dominating set of $T$. Since $T$ is a tree, $2 r(T)-1 \leq d(T) \leq 2 r(T)$.

Suppose that $2 r(T)-1=d(T)$. In this case, any diametral path in $T$ is an even path and $T$ has a bi-center (the center is a pair of adjacent vertices). Consequently, $\hat{r}(T)=r(T)-1$. Applying Theorem 4 to $T$,

$$
\hat{r}(T)+1=r(T)=\frac{d(T)+1}{2} \leq \frac{3 \gamma(T)-1+1}{2}=\frac{3 \gamma(G)}{2} .
$$

From this we find that,

$$
\gamma(G) \geq \frac{2}{3} \hat{r}(T)+\frac{2}{3}>\frac{2}{3} \hat{r}(T)+\frac{1}{3} .
$$

On the other hand, suppose that $2 r(T)=d(T)$. Now, any diametral path in $T$ is an odd path, $T$ has a unique center vertex, and consequently $\hat{r}(T)=r(T)$. Applying Theorem 4 to $T$,

$$
\hat{r}(T)=r(T)=\frac{d(T)}{2} \leq \frac{3 \gamma(T)-1}{2}=\frac{3 \gamma(G)}{2}-\frac{1}{2} .
$$

From this we find that,

$$
\gamma(G) \geq \frac{2}{3} \hat{r}(T)+\frac{1}{3}
$$


To complete the proof we need to show that $\hat{r}(T) \geq \hat{r}(G)$. That is, that the eccentricity of the center of a graph is at most the eccentricity of the center of one of its spanning trees. To this end, observe that the following chain of inequalities is valid:

$$
\hat{r}(G) \leq r(G) \leq r(T) \leq \hat{r}(T)+1
$$

Suppose that $\hat{r}(G)=\hat{r}(T)+1$. This implies that all of the above are equal. In particular, since $\hat{r}(T)+1=r(T), T$ is a bi-centric tree. Let $\{x, y\}$ be the bi-center of $T$. Moreover, let $d_{G}(p, q)$ denote the distance from $p$ to $q$ in $G$. Since for any vertex $w \in G$,

$$
d_{G}(x, w) \leq d_{T}(x, w) \leq r(T)=r(G)
$$

we conclude that $x$ is also a center vertex of $G$. Similarly, $y$ is also a center vertex of $G$.

Let $z$ be a vertex at eccentric distance from $C(G)$ in $G$, and note that for any $v \in C(G)$,

$$
d_{G}(v, z) \geq \hat{r}(G)=\hat{r}(T)+1
$$

Now, because $x$ is a center vertex of $G$,

$$
r(G)=r(T) \geq d_{T}(x, z) \geq d_{G}(x, z) \geq \hat{r}(G)=r(G)
$$

whence $d_{T}(x, z)=r(T)$. Similarly, $d_{T}(y, z)=r(T)$. However, this is a contradiction because only one of these equations can be true for a bi-centric tree. Hence our supposition, $\hat{r}(G)=\hat{r}(T)+1$, is not feasible and it must be the case that $\hat{r}(G) \leq \hat{r}(T)$, proving our claim and thereby proving the theorem.

To see that the bound is sharp, notice that equality holds for paths whose orders are congruent to 3 modulo 6 . On the other hand, for cycles, $\hat{r}(G)=0$ showing that the difference can be made arbitrarily large.

The theorem below is sometimes a substantial improvement on Theorems 4, 5, and 8 . For instance, take a path on $2 k+1$ vertices and amalgamate an endpoint of a path on $k$ vertices to the center vertex of the original path. This graph has $d=2 k, r=\hat{r}=k$, and $\hat{d}=2 k-1$. Thus, the difference between the lower bound obtained below and those bounds obtained from the theorems mentioned above can be made arbitrarily large. On the other hand, for odd paths, the lower bound below can be made arbitrarily less than that obtained from any of the theorems mentioned above. 
Theorem 9. Let $T$ be a tree with $n>1$. Then,

$$
\gamma(T) \geq \frac{1+\hat{d}(T)}{2}
$$

Moreover, this bound is sharp.

Proof. Let $v_{e}$ a vertex of maximum eccentricity from the boundary of $T$. Let $d$ be the diameter of $T$, and $P_{d}=\left\{v_{0}, v_{1}, \ldots, v_{d}\right\}$ be the vertices of a diametral path of $T$ labeled left to right such that $\hat{d}(T)=d_{G}\left(v_{0}, v_{e}\right) \leq d_{G}\left(v_{d}, v_{e}\right)$. If $v_{e}$ is on the diametral path $P_{d}$, then $\hat{d}(T) \leq \frac{d}{2}$, and when $d \geq 2$ the result follows from Theorem 4 . In case $d=1$, the result is trivial. Thus, assume that $v_{e}$ is not on $P_{d}$. Let $P_{e}$ be a path from $v_{0}$ to $v_{e}$. Let $l$ be the largest subscript such that $v_{l}$ is common to both $P_{e}$ and $P_{d}$. Observe that since $T$ is a tree and $d_{G}\left(v_{0}, v_{e}\right) \leq d_{G}\left(v_{d}, v_{e}\right), \frac{d}{2} \geq l \geq 1$.

Let $T^{\prime}$ be the subtree induced by $P_{e}$ and $P_{d}$. The rest of this proof consists of showing that $\frac{1}{2}(1+\hat{d}(T)) \leq \gamma\left(T^{\prime}\right)$ from which the full result follows since it is straightforward to argue that $\gamma\left(T^{\prime}\right) \leq \gamma(T)$. Observe that the number of vertices of $T^{\prime}$ is at least $1+\hat{d}(T)+$ $d-l$. Combining this observation with $l \leq \frac{d}{2}$, it follows that

$$
1+\hat{d}(T)+\frac{d}{2} \leq n\left(T^{\prime}\right)
$$

Since eccentricity of the boundary is strictly less than the diameter, it follows that

$$
\frac{3}{2}(1+\hat{d}(T)) \leq n\left(T^{\prime}\right)
$$

For $D$ a smallest dominating set of $T^{\prime}$ that contains no leaves, let $k$ be the number of components of the subgraph induced by $D$, let $j$ be the number components of the subgraph induced by $T^{\prime}-D$ with at least two neighbors in $D$, and $l_{H}$ be the number components of $T^{\prime}-D$ with exactly one neighbor in $D$. By Lemma 2 parts a), b) and d), the number of edges of $T^{\prime}$ is bounded above as follows,

$$
\frac{3}{2}(1+\hat{d}(T))-1 \leq n\left(T^{\prime}\right)-1 \leq\left[\gamma\left(T^{\prime}\right)-k\right]+\left[k-1-O_{d}\right]+\left[k+j+l_{H}-1\right],
$$

where $O_{d}$ is the number of vertices of $T^{\prime}-D$ that are over-dominated by $D$. Since $T^{\prime}$ has 3 leaves and $D$ contains no leaves of $T^{\prime}, l_{H} \geq 3$. It is not difficult to show that any component of $T^{\prime}-D$ that is not a leaf of $T^{\prime}$ must have two neighbors in $D$, therefore $l_{H}=3$. Now the above relation is equivalent to

$$
\frac{3}{2}(1+\hat{d}(T)) \leq n\left(T^{\prime}\right) \leq \gamma\left(T^{\prime}\right)-O_{d}+k+j+2 .
$$


It is obvious that $k \leq \gamma\left(T^{\prime}\right)$, and by Lemma 2 part $\left.\mathrm{d}\right), j \leq k-1$. So, it follows that

$$
\frac{3}{2}(1+\hat{d}(T)) \leq n\left(T^{\prime}\right) \leq \gamma\left(T^{\prime}\right)-O_{d}+2 k+1 \leq 3 \gamma\left(T^{\prime}\right)+1
$$

In the case that $\frac{3}{2}(1+\hat{d}(T))<n\left(T^{\prime}\right)$ or $O_{d} \geq 1$, we see that

$$
\frac{1}{2}(1+\hat{d}(T)) \leq \gamma\left(T^{\prime}\right)
$$

Thus, we now assume that $\frac{3}{2}(1+\hat{d}(T))=n\left(T^{\prime}\right)$ and that $O_{d}=0$ (i.e. there are no over-dominated vertices in $\left.T^{\prime}-D\right)$. These assumptions together with relation (1) imply,

$$
\frac{3}{2}(1+\hat{d}(T))=n\left(T^{\prime}\right) \leq \gamma\left(T^{\prime}\right)+k+j+2 .
$$

Suppose that $v_{l}$ is not in $D$. Since there are no over-dominated vertices in $T^{\prime}-D$, the component in $T^{\prime}-D$ containing $v_{l}$ has at least 3 vertices and thus contributes 3 edges to $e_{3}$, where $e_{3}$ is the number of edges with one vertex in $D$ and one in $T^{\prime}-D$. Since the remaining $j-1$ non-trivial components of $T^{\prime}-D$ contribute at least two edges to $e_{3}$, $2(j-1)+3+l_{H} \leq e_{3}$. Together with the right hand side of part $\left.\mathrm{d}\right)$ of Lemma 2 this yields, $j \leq k-2$, which together with relation (2) yields the desired result.

To complete our proof we assume that $v_{l}$ is in $D$. In this case, let $P_{1}$ be the vertices $\left\{v_{0}, v_{1}, \ldots, v_{l-1}\right\}$ on the path $P_{d}$, let $P_{3}$ be the vertices $\left\{v_{l+1}, v_{l+2}, \ldots, v_{d}\right\}$ on the path $P_{d}$ and let $P_{2}$ be the remaining vertices of $T^{\prime}$ (this includes $v_{l}$ and the vertices on path $P_{e}$ that are not on $\left.P_{d}\right)$. Since $\left|P_{1}\right|+\left|P_{2}\right|+\left|P_{3}\right|=\frac{3}{2}(1+\hat{d}(T))=n\left(T^{\prime}\right)$ and $\left|P_{1}\right|+\left|P_{2}\right|=1+\hat{d}(T)$, $\left|P_{3}\right|=\frac{1}{2}(1+\hat{d}(T))$. Observe that $\left|P_{1}\right| \leq\left|P_{3}\right|$, otherwise we contradict that vertex $v_{d}$ is not closer to $v_{e}$ than is $v_{0}$. Also observe that $\left|P_{2}\right| \leq\left|P_{1}\right|$, otherwise the path induced by $P_{2} \cup P_{3}$ is longer than a diametral path. Thus, it follows each of $P_{1}, P_{2}$ and $P_{3}$ has exactly $\frac{1}{2}(\hat{d}(T)+1)$ vertices.

Now observe that if $k<\gamma\left(T^{\prime}\right)$, then together with $j \leq k-1$ and inequality (2) the result follows. So we assume $k=\gamma\left(T^{\prime}\right)$ (that is $D$ induces an empty subgraph). Now since we have assumed that $v_{l}$ in $D$, none of the neighbors of $v_{l}$ are in $D$. Since the number of vertices on paths $P_{1}$ and $P_{2}$ not dominated by $v_{l}$ differ by 1 , it is easy to see that at least one of the paths will contain a vertex that is over-dominated. This contradicts our assumption that there are no over-dominated vertices.

To see that this bound is sharp, notice that equality holds for odd paths of order $2 k+1$ with a path of order $k$ amalgamated at the center vertex. On the other hand, odd paths 
show that the difference between the two sides of the inequality can be made arbitrarily large.

Although Theorem 9 was proven only for trees, we conjecture that it is true for all simple connected graphs. In fact, we believe a similar proof to the one above can be used to show this. To see that there are non-tree graphs for which it is true, notice it trivially holds for all connected graphs for which every vertex is in the boundary set - cycles for instance. Now, let the vertices of a $P_{7}$ be labeled from left to right as $\left\{v_{1}, v_{2}, \ldots, v_{7}\right\}$ and let the vertices of a $P_{3}$ be labeled $\{a, b, c\}$. Add the three edges $\left\{a, v_{3}\right\},\left\{b, v_{4}\right\}$, and $\left\{c, v_{5}\right\}$ and then attach a pendant vertex to $b$. This is a non-trivial example of a non-tree graph for which equality holds in the conjecture below.

Conjecture 10. Let $G$ be a connected graph with $n>1$. Then,

$$
\gamma(G) \geq \frac{1+\hat{d}(G)}{2}
$$

Given a graph $G$, the girth, denoted $g=g(G)$, is the minimum order of an induced cycle of $G$.

Theorem 11. Let $G$ be a connected graph with $n>1$. Then,

$$
\gamma \geq \frac{1}{3} g
$$

Moreover, this bound is sharp.

Proof. The inequality is obviously true when $g \leq 3$ so we can assume $g \geq 4$. Let $D$ be a minimum dominating set and let $C$ be an induced cycle of order $g$. Moreover, let $K=D \cap C$. Without loss of generality, $|K|<\frac{g}{3}$ (the inequality is trivial otherwise). Since each vertex of $K$ dominates three vertices of $C$, at most $3|K|$ vertices of $C$ are dominated by vertices from $K$. This leaves at most $g-3|K|$ vertices of $C$ which are not dominated by $K$. Finally, since no two of these un-dominated vertices of $C$ could be adjacent to the same vertex of $D-K$ or a shorter cycle is present, we find;

$$
\gamma \geq|K|+(g-3|K|)=g-2|K|>\frac{1}{3} g,
$$

To see that the bound is sharp, notice that equality holds for all cycles with orders congruent to 0 modulo 6 . On the other hand, the difference can be made arbitrarily large by amalgamating paths to the vertices of a triangle. 
The case of equality for Theorem 11 is not hard to discover. For $g \leq 3$, equality holds if and only if $G$ has a vertex of degree $n-1$ and at least one triangle.

For $4 \leq g \leq 6$, equality holds if and only if: 1) $G$ consists of an induced hexagon, together with a collection of vertices $S$ not on the hexagon, such that every vertex of $S$ is adjacent to one of a pair $\{x, y\}$ of diametrically opposed vertices of the hexagon. 2) Each vertex of $N(x) \cap S$ (resp. $N(y) \cap S$ ) is adjacent to at most one vertex of $N(y) \cap S$ (resp. $N(x) \cap S)$ and no other vertices of $G$.

For $g \geq 7$, equality holds if and only if $g \equiv 0(\bmod 3)$ and $G$ consists of a cycle of order $g$ whose vertices can be labeled clockwise $1,2, \ldots, g$ such that all non-cycle vertices are pendants and are adjacent to vertices of the cycle whose labels come from the same congruence class mod 3 .

Recall that a matching is an independent set of edges. While much is known about the cardinality of maximum matchings, [21], and in particular, that this number can be computed in polynomial time, the problem of determining the order of a maximal matching of minimum size is NP-complete [16]. In fact, this problem remains NP-complete even when restricted to bipartite graphs.

Theorem 12. Let $T$ be a tree where $\mu^{*}$ is the cardinality of a maximal matching of minimum size.

$$
\mu^{*} \leq \gamma \leq 2 \mu^{*}
$$

Moreover, these bounds are sharp.

Proof. The upper bound is obvious since the vertices of any maximal matching are a dominating set.

Let $D=\left\{d_{1}, d_{2}, \ldots, d_{\gamma}\right\}$ be a minimum dominating set of $T$ which is labeled so that $d_{i}$ is no further from the center of the tree than $d_{j}$ whenever $i>j$. If $T$ is bi-central, then choose one of these two vertices and call it the official center. Thus, lower indices indicate greater distance from the center. Our strategy is to build a maximal matching and label its edges in such a way that at most $\gamma$ labels are used.

We build the matching $M$ as follows. For each $i$ - unless $d_{i}$ is already incident to an edge in $M-$ add the edge $d_{i} v$, where $v$ is the vertex adjacent to $d_{i}$ and closest to the center, and label this edge $e_{i}$. If the edge $d_{i} v$ is already incident to an edge of $M$, then choose any other edge incident to $d_{i}$, that is not adjacent to an edge of $M$, and add that edge to $M$ - again labeling it $e_{i}$. If neither of these is possible, then either $d_{i}$ is incident 
to an edge of $M$ or every edge incident to $d_{i}$ is already adjacent to an edge of $M$. In this case, ignore that vertex and move on to the next dominating vertex. Repeat this for all dominating vertices of $D$ in sequential order.

Now, if $M$ is maximal, then we are done since $\mu^{*} \leq|M| \leq|D|=\gamma$. Assuming otherwise, suppose $x y$ is an edge independent of $M$ (adjacent to none of the edges of $M$ ). Moreover, without loss of generality, assume $y$ is closer to the center than $x$ (since they cannot be the same distance). In this case, neither $x$ nor $y$ is in $D$ - since otherwise this edge would have been accounted for in the previous step when we built $M$. Now vertex $x$ must have a dominating vertex $d_{j}$ further from the center than $x$. Since the edge $d_{j} x$ was not added to $M$ in the previous step, and $x$ is obviously closer to the center than any other neighbor of $d_{j}$, it must be the case that an edge containing $d_{j}$ and one of its neighbors, other than $x$, was added to $M$ at a previous step. Hence there is no edge in $M$ with label $j$. So we can label the edge $x y$ as $e_{j}$, include it in $M$, and then repeat this for any other edge independent of $M$. Eventually, $M$ is maximal and at most $\gamma$ labels have been used to label its edges.

To see that these bounds are sharp, let $T$ be a path with order $n$ such that $n \equiv 0(\bmod$ 3). Then $\gamma=\mu^{*}=\frac{n}{3}$. On the other hand, let $T$ be a tree obtained by attaching a pendant vertex to each of the $n$ vertices of an even path. Here, $\gamma=2 \mu^{*}=\frac{n}{2}$. Thus, equality can hold for both upper and lower bounds and the difference between the domination number and both bounds can be made arbitrarily large.

This theorem is not true for graphs in general. For instance, take two identical stars and add an edge between each pair of corresponding leaves. This family of graphs has $\gamma=2$ while $\mu^{*}$ can be made arbitrarily large.

\section{REFERENCES}

[1] J.A. Bondy and U.S.R. Murty, "Graph Theory With Applications," American Elsevier Publishing, 1976.

[2] F. Buckley and F. Harary, "Distance in Graphs", Addison-Wesley, 1990.

[3] M. Chellali and T. Haynes, A Note on the Total Domination Number of a Tree, J. Combin. Math. Combin. Comput., 58 (2006), 189-193.

[4] F. Chung, The average distance is not more than the independence number, J. Graph Theory, 12 (1988), p. 229-235. 
[5] E. Cockayne, R. Dawes and S. Hedetniemi, Total domination in graphs, Networks, 10 (1980), p. 211-219.

[6] P. Dankelmann, Average distance and the independence number, Discrete Appl. Math., 51 (1994), p. 73-83.

[7] P. Dankelmann, Average distance and the domination number, Discrete Appl. Math., 80 (1997), p. 21-35.

[8] E. DeLaViña, Q. Liu, R. Pepper, W. Waller, and D.B. West, Some Conjectures of Graffiti.pc on Total Domination, Congr. Numer., 185(2007), 81-95.

[9] E. DeLaViña, R. Pepper, and W. Waller, A Note on Dominating Sets and Average Distance, Discrete Math., preprint, accepted in 2008.

[10] A. Dobrynin, R. Entringer and I. Gutman, Wiener index of trees: Theory and applications, Acta Applicandae Mathematicae, 66 (2001), p. 211-249.

[11] P. Erdos, M. Saks, and V. Sos, Maximum Induced Tress in Graphs, J. Graph Theory, 41(1986), 61-79.

[12] S. Fajtlowicz and W. Waller, On two conjectures of Graffiti, Congr. Numer., 55 (1986), p. 51-56.

[13] S. Fajtlowicz, "Written on the Wall" (manuscript), Web address: http://math.uh.edu/s̃iemion.

[14] S. Fajtlowicz, A Characterization of Radius-Critical Graphs, J. Graph Theory, 12(1988), 529-532.

[15] O. Favaron, M. Maheo, and J-F. Sacle, Some Results on Conjectures of Graffiti - 1, Ars Combin., 29(1990), 90-106.

[16] M. Garey and D. Johnson, "Computers and Intractability", W.H. Freeman and Co., New York, Bell Telephone Lab., 1979.

[17] P. Hansen, A. Hertz, R. Kilani, O. Marcotte and D. Schindl, Average distance and maximum induced forest, pre-print, 2007.

[18] T. Haynes, S.T. Hedetniemi and P.J. Slater, "Fundamentals of Domination in Graphs," Marcel Decker, Inc., NY, 1998.

[19] T. Haynes, S.T. Hedetniemi and P.J. Slater, "Domination in Graphs: Advanced Topics," Marcel Decker, Inc., NY, 1998.

[20] M. Lemanska, Lower Bound on the Domination Number of a Tree, Discuss. Math. Graph Theory, 24 (2004), 165-170.

[21] L. Lovasz and M.D. Plummer, "Matching Theory", Acedemic Press, New York, 1986.

[22] D.B. West, Open problems column \#23, SIAM Activity Group Newsletter in Discrete Mathematics, 1996.

[23] D.B. West, "Introduction to Graph Theory (2nd ed.)," Prentice-Hall, NJ, 2001. 